\title{
Mini-BEAM Regimen
}

National Cancer Institute

\section{Source}

National Cancer Institute. Mini-BEAM Regimen. NCI Thesaurus. Code C63372.

A dose-reduced variant of the BEAM conditioning regimen used in the transplant setting, consisting of carmustine, etoposide, cytarabine, and melphalan and used for the treatment of relapsed or refractory Hodgkin lymphoma. 